\title{
Comparative analysis of microbial communities during enrichment and isolation of DDT-degrading bacteria by culture-dependent and -independent methods
}

\author{
Beibei Wang a,b ${ }^{\text {, Wuxing Liu }}{ }^{\mathrm{a}, *}$, Xiaoyan Liu ${ }^{\mathrm{a}, \mathrm{b}}$, Ashley E. Franks ${ }^{\mathrm{c}}$, Ying Teng ${ }^{\mathrm{a}}$, Yongming Luo ${ }^{\mathrm{a}, \mathrm{d}}$ \\ a Key Laboratory of Soil Environment and Pollution Remediation, Institute of Soil Science, Chinese Academy of Sciences, Nanjing 210008, China \\ b University of Chinese Academy of Sciences, Beijing 100049, China \\ c Department of Physiology, Anatomy and Microbiology, La Trobe University, Melbourne, Victoria 3086, Australia \\ d Institute of Coastal Zone Research, Chinese Academy of Sciences, Yantai 264003, China
}

\section{H I G H L I G H T S}

- The isolate, Streptomyces sp. D3, was capable of degrading DDT, PCB77and PCNB.

- Culture-dependent and -independent provide two extremely distinct views.

- Pseudomonas, the frequently isolated only represented the 12th most abundant OTU.

- The most abundant bacterial OTU Pseudorhodoferax was observed by sequencing.

\section{G R A P H I C A L A B S T R A C T}

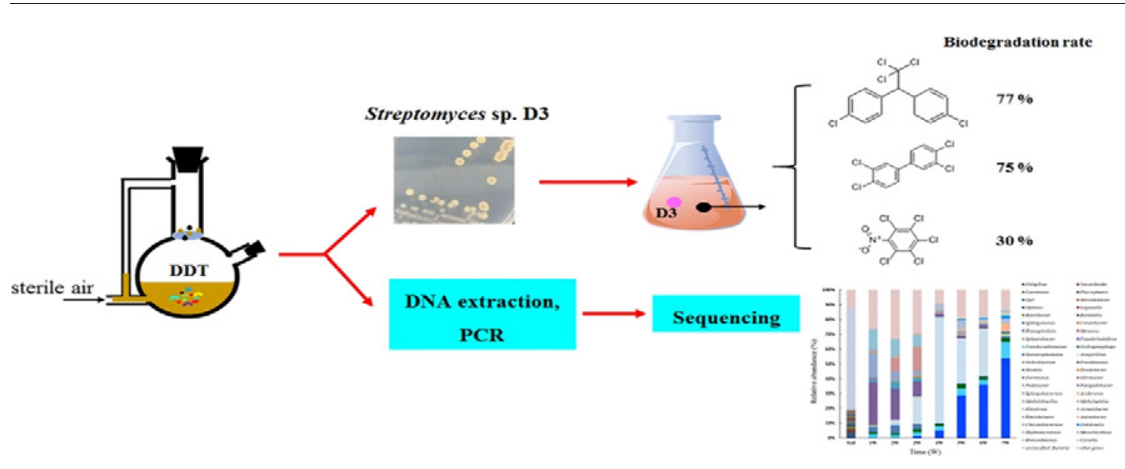

\section{A B S T R A C T}

Microcosms for enrichment of DDT degrading microorganisms were monitored using culture-dependent and -independent methods. Culture dependent methods isolated several strains with DDT degradation potential, Pseudomonas species being the most frequent. One isolate, Streptomyces sp. strain D3, had a degradation rate of $77 \%$ with $20 \mathrm{mg} \mathrm{L}^{-1}$ of DDT after 7 days incubation, D3 also had degradation rates of $75 \%$ and $30 \%$ for PCB77 (3,3',4,4'-tetrachloro biphenyl) and PCNB (pentachloronitrobenzene) respectively. Culture-independent highthroughput sequencing identified a different subset of the microbial community within the enrichment microcosms to the culture dependent method. Pseudomonas, the most frequently isolated strain, only represented the 12 th most abundant operational taxonomic unit in the sequencing dataset (relative abundance $0.9 \%$ ). The most frequently observed bacterial genus in the culture-independent analysis did not correspond with those recovered by culture-dependent methods. These results suggested that deep sequencing followed by a targeted isolation approach might provide an advantageous route to bioremediation studies.

(C) 2017 Elsevier B.V. All rights reserved.

\footnotetext{
* Corresponding author.

E-mail address: liuwuxin@issas.ac.cn (W. Liu).
}

\section{Introduction}

The organochlorine 1,1,1-trichloro-2,2-bis(4-chlorophenyl) ethane (DDT) is a colorless, tasteless and an almost odorless compound that was the most extensively used insecticide globally until the 1960s 
(Bolan, 2012; Simonich and Hites, 1995; Sudharshan et al., 2012). DDT was the first synthesized insecticide, enabled large agricultural production increases, as well as the control of mosquito-borne malaria and typhus (Kim et al., 2016). However, the toxicity and persistence of DDT in the environment has led an almost international ban of its use (World Health Organization, 1989). In the environment, DDTs residues are comprised of the DDT isomers p, $\mathrm{p}^{\prime}$-DDT and o,p'-DDT, as well as the DDT degradation products DDE (2,2-bis(p-chlorophenyl)-1,1-dichlorethylene) and DDD (1,1-dichloro-2,2-bis(p-chlorophenyl)ethane) (Wang et al., 2016a). Even though the use of DDT has been banned since 1972 in the United States and 1983 in China, respectively, DDTs still pose an environmental risk due to persistence (Kamanavalli and Ninnekar, 2004). In some regions of China, the DDTs concentration in soils still exceed soil quality standards due to previous accumulation and slow degradation (Feng et al., 2003; Shi et al., 2005; Zhong et al., 2011). DDTs also continue to accumulate in the food chain and has been found in human adipose and breast milk (Dimitriadou et al., 2016). Other man-made organochlorine compounds, such as polychlorinated biphenyls (PCBs) and pentachloronitrobenzene (PCNB), also persist in soils and have become serious environmental problems (Hu et al., 2008; Li and Yang, 2013). Thus, degradation enhancement strategies of organochlorine compounds in general and DDT specifically is still of great importance.

Investigations have demonstrated that bioremediation is a feasible solution for treating organic contaminants including PCB, PCNB, DDT, DDE and DDD (Fang et al., 2010; Li and Yang, 2013; Liu et al., 2010; Wang et al., 2016a). High efficiency degradation strains are a key factor for bioremediation of organic pollutants. Various genetic engineering approaches have developed and constructed bacteria relevant for biodegradation (Pieper and Reineke, 2000). Molecular engineering has been utilized to enhance the level of a specific protein, enzyme or metabolic pathways in the target bacteria with the goal of increasing degradation rates (Åhman et al., 2002). However, the application of genetically engineered strains is limited by environmental suitability, biosecurity and public acceptance (Sivasubramaniam and Franks, 2016). Therefore, promotion of degradation by indigenous strains isolated from soil is still one of the principal means for successful bioremediation of organochlorine compounds. In the past decades, researchers have isolated strains such as Alcaligenes eutrophus A5, Pseudomonas sp., Pseudoxanthomonas jiangsuensis that could degrade DDT and associate metabolites in culture (Kamanavalli and Ninnekar, 2004; Nadeau et al., 1994; Wang et al., 2011). The process of isolation of DDT degrading bacteria based on traditional culture-dependent methods include enrichment cultures with pollutants as carbon and/or energy sources, followed by separation and purification with selective plating medium. However, this method is generally less targeted and limited due to the complex microbial interactions in soil ecosystems, lack of understanding for microflora and inability to cultivate a majority of the bacteria from the natural environment in the laboratory (Giovannoni et al., 1990; Hugenholtz et al., 1998).

The rapid development of high-throughput sequencing technology has enabled a comprehensive culture independent evaluation of the microbial community composition in complex environments (Andersson et al., 2010). For instance, high-throughput sequencing has been utilized for the investigation of microbial community structure in depth and in various environmental samples including: marine water; soil; human distal intestine and; wastewater treatment plants (Claesson et al., 2009; Qian et al., 2011; Roesch et al., 2007; Ye and Zhang, 2013). Compared with culture-dependent methods, culture-independent methods based on community DNA analysis and include a significantly larger fraction of microorganisms from the environment (Vaz-Moreira et al., 2011). In recent years, some studies have compared the gap between culture-dependent and culture-independent of microbial communities. Stefani et al. (2015) paired culture-independent with culturedependent techniques to analyze the bacterial and fungal communities from hydrocarbon-contaminated soils. None of the bacteria isolated using several types of culturing media were representative of the major bacterial operational taxonomic units (OTUs) recovered by 454pyrosequencing. Similar results were also observed in fresh water sample, soil samples and Montasio cheese manufacturing (Carraro et al., 2011; Vaz-Moreira et al., 2011; Shade et al., 2012). While high-throughput sequencing has typically been applied to analyze microbial communities in the environment, community enrichment during the isolation of contaminate degrading strains is seldom reported.

In order to gain a comprehensive community overview during the enrichment for DDTs degraders, with the goal of improving strain isolation in the future, a culture-independent high-throughput sequencing (Illumina sequencing) was used to investigate the microbial community in depth. An extensive understand of the microbial community composition during enrichment has the potential to guide the isolation of degradation strains by traditional culture-dependent methods and thus improve outcomes. To the best of our knowledge, this is the first study to compare the structure and dynamics of microbial communities during the process of enrichment cultures using high-throughput sequencing, reporting the gap between culture-independent and culturedependent analyses of microbial communities within enrichment cultures.

\section{Materials and methods}

\subsection{Chemicals and growth medium}

DDT and DTT standard solutions were purchased from East Chemical Co., Ltd., Taixing, Jiangsu China and J\&K Scientific Ltd., Beijing, China, respectively. All organic solvents and chemicals were chromatographic or analytical grade.

Yeast mineral salt medium (YMM) was adapted from Xie et al. (2011). Briefly, YMM medium contained $4.0 \mathrm{~g} \mathrm{NaNO}_{3}, 1.5 \mathrm{~g} \mathrm{KH}_{2} \mathrm{PO}_{4}$, $0.5 \mathrm{~g} \mathrm{Na}_{2} \mathrm{HPO}_{4}, 0.01 \mathrm{~g} \mathrm{CaCl}_{2}, 1 \mathrm{mg} \mathrm{FeSO}{ }_{4} \cdot 7 \mathrm{H}_{2} \mathrm{O}, 0.04 \mathrm{~g}$ Yeast extract and $100 \mu \mathrm{L}$ trace element solution per liter, adjusted to a pH 7.0 and autoclaved at $121^{\circ} \mathrm{C}$ for $20 \mathrm{~min}$, DDT dissolved in acetone and sterilized by filtration was supplemented in YMM with concentration of $20 \mathrm{mg} \mathrm{L}^{-1}$. Solid medium was prepared using $2 \%$ agar. Trace element solution contains $10 \mathrm{mg} \mathrm{H} \mathrm{BO}_{3}, 78.2 \mathrm{mg} \mathrm{CuSO}$, $11.2 \mathrm{mg} \mathrm{MnSO}_{4}$, $10 \mathrm{mg} \mathrm{MoO}_{3}$, and $124.6 \mathrm{mg} \mathrm{ZnSO}_{4}$ per $100 \mathrm{~mL}$. Luria-Bertani (LB) medium (Bertani, 1954) was used for strain cultivation and for the measurement of degradation ability of DDT degrading isolates.

\subsection{Enrichment and isolation of DDT-degrading microorganisms}

A soil-charcoal perfusion apparatus was used to create microcosms for the enrichment of DDT-degrading microorganisms from long-term DDT contaminated soil sample as previously describe (Takagi et al., 2009). Charcoal (3.0 g) was mixed with wet soil sample (3.0 g) and placed on a glass filter placed in the neck of the perfusion apparatus. An air pump was used to circulate $200 \mathrm{~mL}$ of sterile YMM supplemented with $20 \mathrm{mg} \mathrm{L}^{-1}$ DDT at $25^{\circ} \mathrm{C}$ as previously described (Harada et al., 2010). Fresh perfusion fluid (YMM supplemented with $20 \mathrm{mg} \mathrm{L}^{-1}$ DDT) was preserved as controls. The perfusion fluid in the apparatus was substituted with fresh medium at a weekly interval. After 7 weeks, a mixed microbial population community was considered to be established when DDT degradation reached a steady state.

At steady state, a piece of charcoal was removed from the perfusion apparatus, crushed in perfusion fluid $(1 \mathrm{~mL})$ and added into sterile water $(9 \mathrm{~mL})$. Serial dilutions of the suspension was prepared $\left(10^{-1}\right.$ $10^{-5}$ ) and $100 \mu \mathrm{L}$ spread plated onto the YMM medium agar supplemented with $20 \mathrm{mg} \mathrm{L}^{-1}$ of DDT as the energy and carbon source. After incubation for $48 \mathrm{~h}$ at $30^{\circ} \mathrm{C}$, individual colonies of distinct morphology were isolated through streak plating on the YMM medium supplemented with $20 \mathrm{mg} \mathrm{L}^{-1}$ of DDT. 


\subsection{Identification of isolated strains}

DNA was extracted from purified cultures utilizing the bacterial genomic DNA Extraction Kit (centrifugal column type, GeneRay Technology) as per the manufactures instructions. The 16S rRNA gene was amplified using primers $8 \mathrm{~F}$ ( $5^{\prime}$-AGAGTTTGATCCTGGCTCAG-3') (Eden et al., 1991) and 1492R (5'-TACCTTGTTACGACTT-3') (Jiang et al., 2006). Amplifications were carried out in $50 \mu \mathrm{L}$ reaction mixture, which contained $25 \mu \mathrm{L}$ Premix Taq $^{\mathrm{TM}}$ (a $2 \times$ mixture solution of buffer, DNA polymerase and dNTPs), $1 \mu \mathrm{L}$ of each primer (10 mM), $1 \mu \mathrm{L}$ DNA template $\left(20 \mathrm{ng} \mu \mathrm{L}^{-1}\right.$ ) and $22 \mu \mathrm{L} \mathrm{ddH_{2 }} \mathrm{O}$. The amplification cycle: initial denaturation $\left(95^{\circ} \mathrm{C} 300 \mathrm{~s}\right)$ followed by 30 cycles of denaturation $\left(95^{\circ} \mathrm{C}\right.$ $30 \mathrm{~s}$ ), annealing $\left(55^{\circ} \mathrm{C} 30 \mathrm{~s}\right)$ and extensions $\left(72{ }^{\circ} \mathrm{C} 30 \mathrm{~s}\right.$ ) before a final extension $\left(72^{\circ} \mathrm{C} 300 \mathrm{~s}\right)$. Amplified replicate reaction mixes were pooled for purification and analyzed by DNA electrophoresis (1.8\% Agarose gel, $120 \mathrm{~V}$ for $30 \mathrm{~min}$ ). Amplified product was excised from gel, cleaned up using AxyPrep DNA Gel Extraction Kit (Axygen Biosciences) and sequenced by Majorbio Bio-pharm Technology Co., Ltd. (Shanghai China).

\subsection{Monitoring of DDT degradation during enrichment process}

The DDTs concentration in the perfusion fluid was determined weekly by gas chromatography. The standard curves for all compounds were prepared with 'external standard' type. The YMM culture solution in the perfusion apparatus was collected and replace with fresh media at specific time points. Organic compounds were extract from $20 \mathrm{~mL}$ enriched culture medium with $40 \mathrm{~mL}$ n-hexane, then filtered and diluted for further testing. The concentration of DDT in enrichment culture solution were analyzed by gas chromatography (Agilent 7890B) equipped with a ${ }^{63} \mathrm{Ni}$ electron capture detector (GC-ECD) and a $30 \mathrm{~m} \times 0.25 \mu \mathrm{m} \times 0.25 \mu \mathrm{m}$ HP-5 capillary column. Ultra-high purity $\mathrm{N}_{2}$ (99.9999\%) was used as the carrier gas. All experiments were conducted in triplicated.

\subsection{Microbial diversity analysis}

Bacterial diversity and abundance during the process of enrichment was investigated through sequencing of the $16 \mathrm{~S}$ ribosomal RNA gene. DNA was extract from the DDT long-term contaminated soil sample used as the original inoculum and weekly from the soil-charcoal perfusion apparatus using FastDNA ${ }^{\mathrm{TM}}$ SPIN Kit (MP Biomedicals) according to manufacturer's protocols. The V4-V5 region of the bacteria $16 \mathrm{~S}$ ribosomal RNA gene was amplified using primers F515 (5'-GTGCCAGC MGCCGCGG-3') and R907 (5'-CCGTCAATTCMTTTRAGTTT-3') (Zhou et al., 2011). Polymerase chain reaction (PCR) reactions were performed in triplicate $50 \mu \mathrm{L}$ mixture containing $0.8 \mu \mathrm{L}$ of each primer $(5 \mu \mathrm{M}), 10 \mathrm{ng}$ of template DNA, $2 \mu \mathrm{L}$ of $2.5 \mathrm{mM}$ dNTPs, $0.4 \mu \mathrm{L}$ of FastPfu Polymerase and $4 \mu \mathrm{L}$ of $5 \times$ FastPfu Buffer. PCR reaction was conducted as follows: initial denaturation $\left(95^{\circ} \mathrm{C} 2 \mathrm{~min}\right.$ ), followed by 25 cycles of denaturation $\left(95^{\circ} \mathrm{C}\right.$ $30 \mathrm{~s})$, annealing $\left(55^{\circ} \mathrm{C} 30 \mathrm{~s}\right)$, and extensions $\left(72{ }^{\circ} \mathrm{C} 30 \mathrm{~s}\right)$ before a final extension $\left(72{ }^{\circ} \mathrm{C} 5 \mathrm{~min}\right)$.

Amplicons were extracted from 2\% agarose gels and purified using the AxyPrep DNA Gel Extraction Kit (Axygen Biosciences, Union City, CA, U.S.). Purified amplicons were pooled in equimolar and paired-end sequenced $(2 \times 250)$ on an Illumina MiSeq platform according to the standard protocols of Majorbio Bio-pharm Technology Co., Ltd. (Shanghai China).

\subsection{Processing of Illumina MiSeq data}

MiSeq sequencing data were analyzed according to Hou et al. (2015). Bacterial sequences were classified using a Ribosomal Database Project naïve Bayesian rRNA classifier (RDP) with a confidence of $80 \%$. At each taxonomic level, relative abundance was calculated as a percent of all sequences classified in that sample. OTUs were classified at similarities of $97 \%$ after normalization to 14,000 sequences per sample.
The a-diversity indices, including observed OTUs, Chao and InvSimpson, were calculated using MOTHUR 1.33.0.

\subsection{Organochlorine compound degradation ability of DDT degrading isolates}

Isolated strains were cultured in LB liquid media at $30{ }^{\circ} \mathrm{C}$ until midexponential phase. Cells were harvested and re-suspended in sterile water to an OD of 1.0 at $600 \mathrm{~nm}$ as an inoculant. Triplicate conical flask containing $20 \mathrm{~mL}$ of YMM supplemented with $20 \mathrm{mg} \mathrm{L}^{-1}$ of DDT were inoculated with $1 \mathrm{~mL}$ bacterial suspensions, flask containing media with DDT inoculated with $1 \mathrm{~mL}$ of autoclave sterilized inoculum acted as controls. The samples were cultured in the dark at $30{ }^{\circ} \mathrm{C}$ with shaking for 7 days. The liquid medium culture $(20 \mathrm{~mL})$ was extracted with $40 \mathrm{~mL}$ hexane by shaking vigorously. The organic extract was dried over anhydrous sodium sulfate and then diluted gradient for GCECD analysis. The strain with highest degradation efficiency, as determined by the degradation rate, was selected for further study.

Bacterial suspensions with highest DDT degradation efficiency, prepared as above, inoculated into YMM media in triplicate containing with $20 \mathrm{mg} \mathrm{L}^{-1}$ of PCB 77 or $40 \mathrm{mg} \mathrm{L}^{-1}$ PCNB and cultured in the dark at $30{ }^{\circ} \mathrm{C}$ with shaking, uninoculated YMM media with PCB77 or PCNB was utilized as control. After 7 days, PCB77 and PCNB was extracted as per DDT, and concentrations determined by GC-ECD.

The oven temperature program for DDT was as follows: injector and detector temperatures $200{ }^{\circ} \mathrm{C}$ and $300{ }^{\circ} \mathrm{C}$ respectively, initial run temperature $80{ }^{\circ} \mathrm{C}$, ramped at $50{ }^{\circ} \mathrm{C} \mathrm{min}-1$ to $210{ }^{\circ} \mathrm{C}$, then ramped at $1{ }^{\circ} \mathrm{C} \mathrm{min}^{-1}$ to $220^{\circ} \mathrm{C}$ and held for $1 \mathrm{~min}$, and finally ramped at $2{ }^{\circ} \mathrm{C} \mathrm{min}^{-1}$ to $230{ }^{\circ} \mathrm{C}$. Split injection was applied ( $\left.1 \mu \mathrm{L}, 10: 1\right)$. The oven temperature program for PCNB was as follows: injector and detector temperatures $260^{\circ} \mathrm{C}$ and $310^{\circ} \mathrm{C}$ respectively, initial run temperature $80^{\circ} \mathrm{C}$ and held for $0.5 \mathrm{~min}$, ramped at $40^{\circ} \mathrm{C} \mathrm{min}^{-1}$ to $210^{\circ} \mathrm{C}$ and held for $0.5 \mathrm{~min}$, ramped at $6{ }^{\circ} \mathrm{C} \mathrm{min}^{-1}$ to $230^{\circ} \mathrm{C}$ and held for $1 \mathrm{~min}$, finally ramped at $40^{\circ} \mathrm{C} \mathrm{min}^{-1}$ to $280^{\circ} \mathrm{C}$ and held for $0.5 \mathrm{~min}$. The oven temperature program for PCB77 was conducted according to Wang et al. (2016b).

The degradation rate was calculated according to the following equation: Degradation rate $(\%)=\left(C_{0}-C\right) / C_{0} \times 100 \%$, where $C_{0}$ and $\mathrm{C}$ are the initial and residual concentration of organochlorine compounds at the time indicated, respectively.

\subsection{Data analysis}

Statistical analysis was conducted with SPSS 17.0 software. Duncan's multiple range tests were used to compare the means of treatments, variability in the data was expressed as the standard errors. All analyses were performed at the $p<0.05$ level.

\subsection{Nucleotide sequence accession number}

The isolated strain Streptomyces sp. D3 was submitted to GenBank, accession number KX762051. The strain is deposited in the Chinese Culture Collection Management Committee General Microbiology Center (CGMCC), accession number is CGMCC No.12904. The Illumina sequencing data was submitted to the NCBI Sequence Read Archive database, SRA accession numbers are SRR4162049 for soil, SRR4162050SRR4162056 for enrichment culture of every week.

\section{Results}

\subsection{Monitoring of DDT degradation during enrichment process}

After being refreshed with YMM containing DDT at $20 \mathrm{mg} \mathrm{L}^{-1}$, the concentration of DDTs (including DDT, DDE and DDD) decreased and the corresponding degradation rate increased each week. A a gradually increase in the degradation of DDT was observed over the 7 week period 

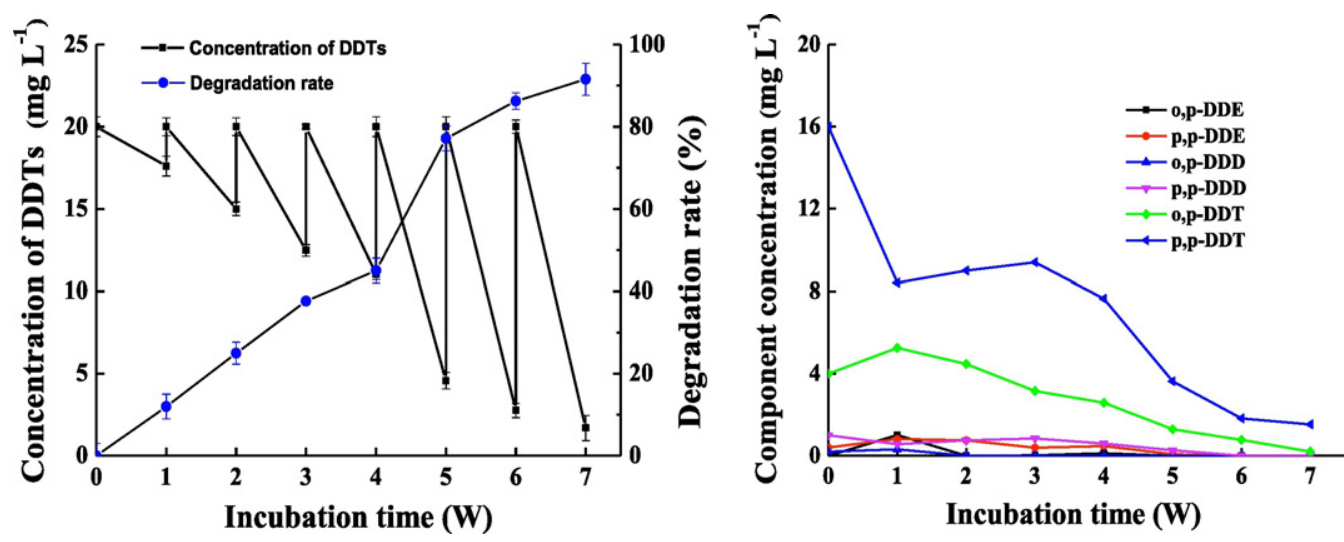

Fig. 1. The variation of concentration for DDTs in the enrichment process over 7 weeks.

(Fig. 1). In the first four weeks, the DDTs degradation increased from $10 \%$ to $45 \%$ at a relatively steady rate. At the end of 7 weeks, the degradation rate of DDTs reached $92 \%$.

\subsection{Enrichment and isolation of DDT-degrading bacteria}

DDT degrading microorganisms enriched within the microcosm were cultured with selective media from the charcoal and the culture solution. In this study, 24 strains designated D1-D24 were identified and grouped into 12 different species with DDT degradation ability ranging from 0.1 to $77.3 \%$ (Table 1 ). Individual degradation rates were lower than the mixed community enrichment culture (92\%). According to traditional culture-dependent methods, Pseudomonas and Phyllobacterium species were predominant after 7 weeks $(16.7 \%$ among the isolates), but the maximum DDTs degradation ability was only $25 \%$ and $10 \%$, respectively. However, Streptomyces sp. D3 showed the highest degradation ability of DDTs (77\%). The DDT degradation ability of isolates was lower than that of the mixed community in the apparatus at the end of 7 weeks (92\%). This phenomenon may be attribute to synergistic effect between the species in mixed culture.

\subsection{The dynamic changes at the community level}

High throughput sequencing of the perfusion apparatus during enrichment allowed in depth analysis of the microbial community. After processing of raw sequence data, a total of 159,093 sequences were analyzed. Samples ranged from 14,309 to 25,546 OTUs classified at similarities of $97 \%$ after normalized to 14,000 sequences per sample (Table 2). The diversity indices, including OTUs, Chao and InvSimpson, were highest in the original soil inoculum and showed a decrease

Table 1

Identification result of isolated strains based on the $16 \mathrm{~S}$ rRNA gene sequences analysis and efficiency of DDTs degradation.

\begin{tabular}{llll}
\hline Number of strains & Identification result & $\begin{array}{l}\text { Percentage } \\
(\%)\end{array}$ & $\begin{array}{l}\text { Degradation rate } \\
(\%)\end{array}$ \\
\hline D16, D17, D9, D11 & Pseudomonas sp. & 16.7 & $7.1-25.3$ \\
D19, D20, D21, D24 & Phyllobacterium sp. & 16.7 & $5.3-13.6$ \\
D15, D6, D12 & Aminobacter sp. & 12.5 & $12.7-17.0$ \\
D2, D7, D14 & Pseudoxanthomonas sp. & 12.5 & $14.9-23.4$ \\
D1, D4, D5 & Bacillus sp. & 12.5 & $6.7-12.9$ \\
D18 & Sphingobacterium sp. & 4.2 & 22.3 \\
D8 & Paenibacillus sp. & 4.2 & 6.9 \\
D22 & Rhodococcus sp. & 4.2 & 50.2 \\
D23 & Achromobacter sp. & 4.2 & 8.4 \\
D3 & Streptomyces sp. & 4.2 & 77.3 \\
D13 & Ochrobactrum sp. & 4.2 & 0.1 \\
D10 & Variovorax sp. & 4.2 & 1.3 \\
& & &
\end{tabular}

until week 4 before rising slightly in weeks 5,6 and 7 (Table 1). Overall, a decrease in the diversity of the microbial community during the enrichment culture process was observed.

Bacterial sequences were classified into 23 phyla (Eubacteria 21 and Archaea 2). Because of the total reads of Archaea were very low, they were excluded from further analysis in this study. At the end of 7 weeks, Proteobacteria were identified as the most abundant phyla in all samples, of which $\alpha$-proteobacteria and $\gamma$-proteobacteria were preponderant class in soil sample (27\% and $33 \%$ ). However, the abundance of $\alpha$-proteobacteria and $\gamma$-proteobacteria decreased to $14 \%$ and $17 \%$, and the relative abundance of $\beta$-proteobacteria significantly increased from $6 \%$ to $60 \%$ (data not shown). The relative abundances of the top 15 genus in each samples varied over time (Fig. 2). The largest difference was observed between the soil sample and culture medium samples. Relative abundance of Pseudorhodoferax, Pseudoxanthomonas, Dokdonella, Aminobacter and Hydrogenophaga all increased during the experiment, especially, Pseudorhodoferax that reached a relative abundance of $53.8 \%$ after 7 weeks.

\subsection{Comparison of isolated strains and in depth community sequencing}

Pseudorhodoferax became the dominant genus, in terms of relative abundance, reaching $53.8 \%$ at the end of 7 weeks, followed by Pseudoxanthomonas (11\%) and Aminobacter (6\%) (Table 3). Using traditional culture-dependent methods (Table 1), Pseudomonas was the most frequently isolated, but only represented the 12th most abundant OTU in the culture-independent methods dataset. In addition, most of the shared OTUs belong to the genera Pseudoxanthomonas and Aminobacter. However, Pseudorhodoferax, the most frequently observed bacterial genus in the culture-independent sequences, cannot be purified by culture-dependent methods. These results indicated that there are distinctly difference between culture-independent dataset and culture-independent sequencing.

Table 2

The number of sequences and calculated diversity indices for each sample at weekly intervals.

\begin{tabular}{lllll}
\hline Sample & Sequences & OTUs & Chao & Invsimpson \\
\hline Soil & 24697 & 3340 & 24650 & 26.25 \\
1W & 16739 & 2004 & 8939 & 14.93 \\
2W & 21275 & 2340 & 12228 & 24.77 \\
3W & 19966 & 1843 & 8406 & 14.87 \\
4W & 14309 & 811 & 3325 & 2.04 \\
5W & 25546 & 1169 & 5588 & 6.67 \\
6W & 20863 & 1264 & 6505 & 4.95 \\
7W & 15698 & 1174 & 5420 & 3.45 \\
\hline
\end{tabular}

Note: W, week. 


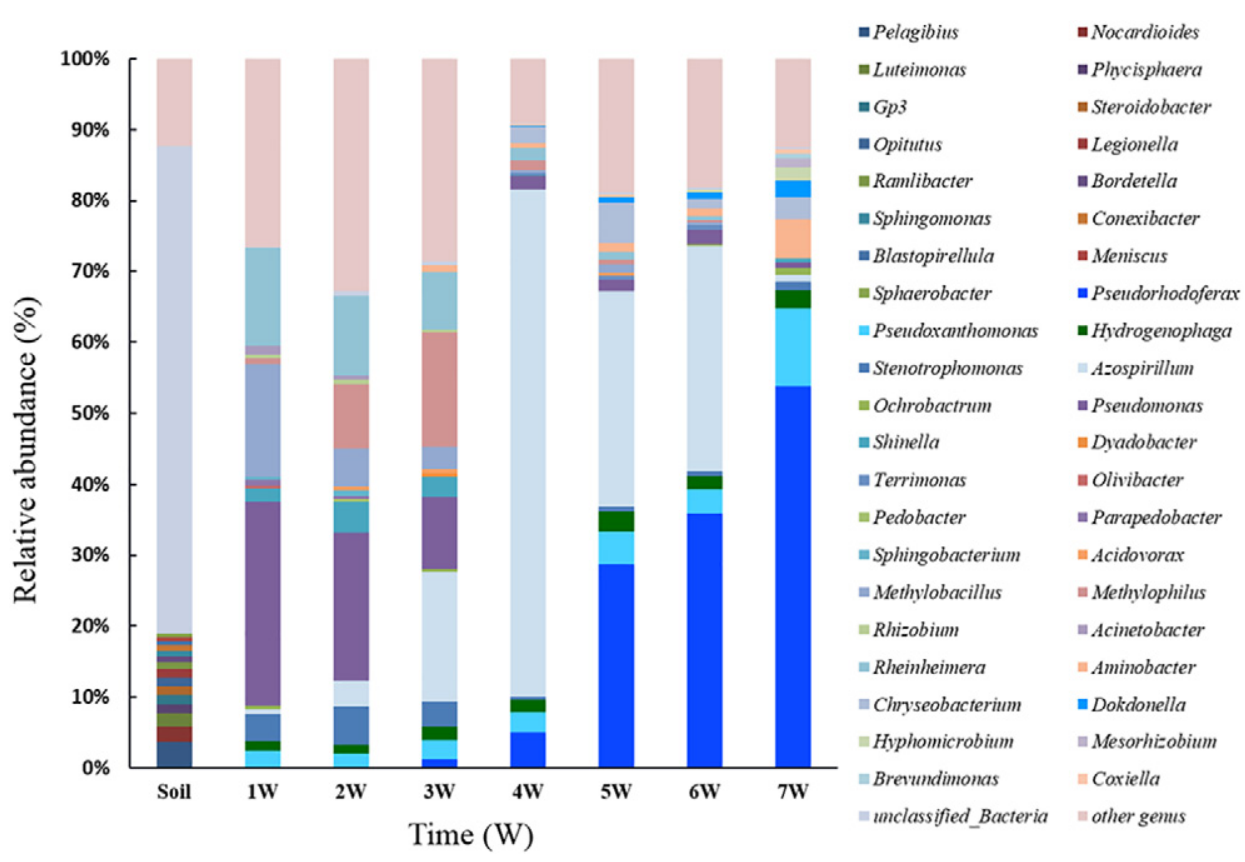

Fig. 2. The relative abundance of bacterial taxa at genus level at weekly time intervals.

\subsection{Ability of isolated strains to degrade DDT and other organochlorine compounds}

Streptomyces sp. D3, with the highest DDT degradation ability, was used for further study. The concentrations of the individual DDT components and DDTs declined after 7 days inoculation with Streptomyces sp. D3 (Fig. 3). The concentrations of individual DDT isomers decreased by $88 \%$ (o,p-DDT) and 60\% (p,p-DDT), resulting in a declined of DDTs from $20.8 \mathrm{mg} \mathrm{L}^{-1}$ (control) to $5.7 \mathrm{mg} \mathrm{L}^{-1}$, with a degradation efficiency of $77 \%$.

The ability to degrade other organochlorine compounds, such as PCB77 and PCNB were also investigated. Streptomyces sp. D3 decreased PCB 77 from $17.9 \mathrm{mg} \mathrm{L}^{-1}$ (control) to $4.4 \mathrm{mg} \mathrm{L}^{-1}$ in 7 days with a degradation efficiency of $75 \%$ and PCNB was decreased from $35.0 \mathrm{mg} \mathrm{L}^{-1}$ (control) to $25.0 \mathrm{mg} \mathrm{L}^{-1}$, with a degradation efficiency of $30 \%$ (Fig. 4). These results showed that D3 could degrade PCB77 and PCNB, in addition to DDT.

\section{Discussion}

Microorganisms are considered to be the primary source of degradation of organic pollutants in natural environment. In the present study,

Table 3

OTUs with relative abundance $>0.5 \%$ at the end of 7 weeks.

\begin{tabular}{lll}
\hline Genus & Sequences & Relative abundance (\%) \\
\hline Pseudorhodoferax & 8448 & 53.8 \\
Pseudoxanthomonas & 1730 & 11.0 \\
Aminobacter & 858 & 5.5 \\
Chryseobacterium & 500 & 3.2 \\
Hydrogenophaga & 405 & 2.6 \\
Dokdonella & 353 & 2.2 \\
Hyphomicrobium & 300 & 1.9 \\
Mesorhizobium & 196 & 1.2 \\
Stenotrophomonas & 183 & 1.2 \\
Azospirillum & 148 & 0.9 \\
Ochrobactrum & 142 & 0.9 \\
Pseudomonas & 135 & 0.9 \\
Brevundimonas & 103 & 0.7 \\
Coxiella & 101 & 0.6 \\
Shinella & 92 & 0.6 \\
\hline
\end{tabular}

DDT-degrading microorganisms were isolated with selective medium using culture-dependent method. However, the isolated strains showed poorer degradation ability than that of the community of enrichment in the apparatus. Microbial consortia are often reported as more efficient and effective for biological degradation of pesticides than the individual strains, due to the range of metabolic and ecosystem level interactions in mixed communities (Verma et al., 2014). In microbial communities, microorganisms cooperate to co-metabolize contaminants that individual microorganism cannot. Kim et al. (2009) observed that the bacteria of three species (Klebsiella sp., Stenotrophomonas sp. and Acinetobacter sp.) could degrade phenylephrine (PHE) more efficient as a mixture. The degradation efficiency in mixed consortia of Acinetobacter sp. and Stenotrophomonas sp. (about $80 \%$ ) was higher than a single microorganism (48.0, 11.0, and 9.0\% for Acinetobacter sp., Klebsiella sp., and Stenotrophomonas sp. respectively). The mixed cultivation of microbes might be effective in bioremediation, even if the microorganisms have low degradation efficiency in pure culture. Since microbial interactions is of great significance in shaping microbial community activity and structure (Cornforth and Foster, 2013; Foster and Bell, 2012), the development of the community is important over time. Therefore, it is

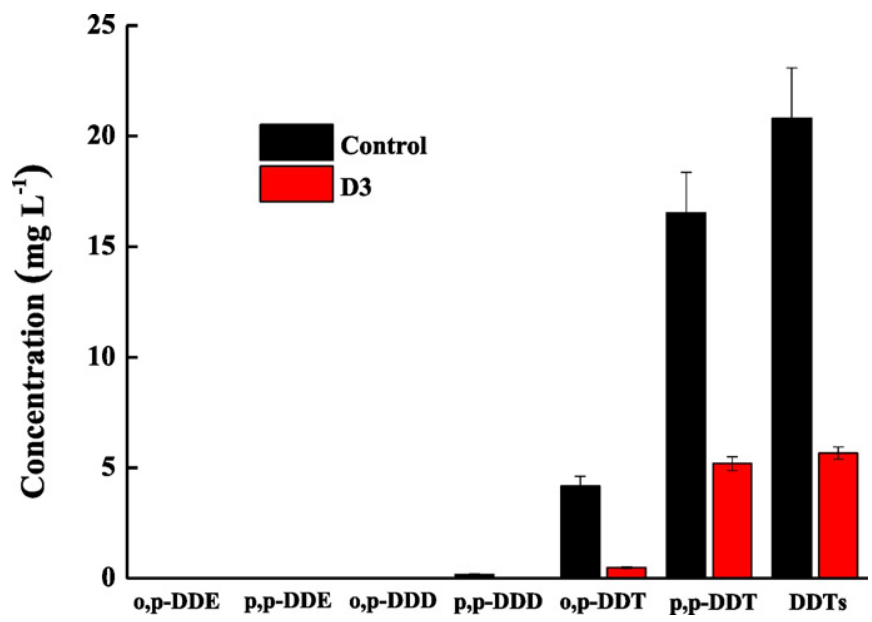

Fig. 3. DDTs degradation ability of Streptomyces sp. D3. 


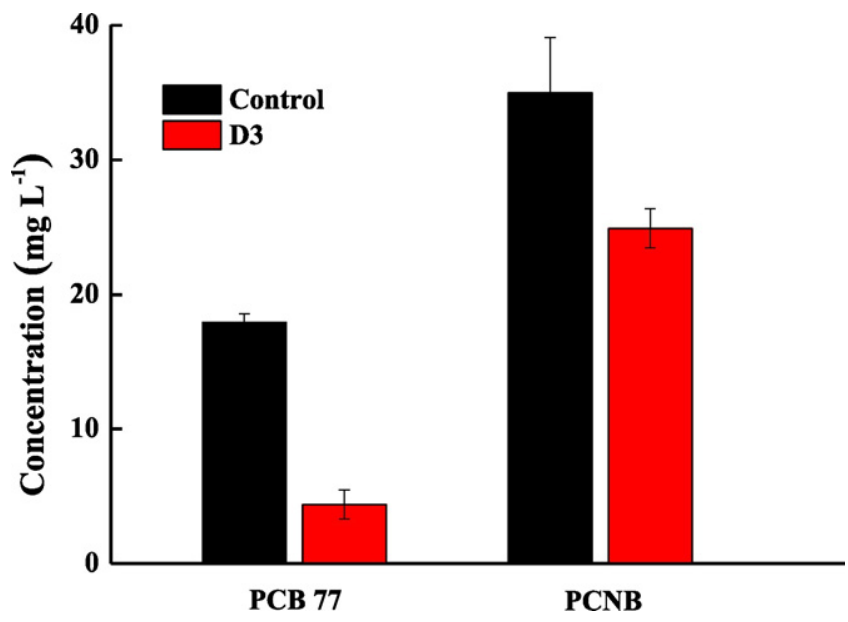

Fig. 4. PCB77 and PCNB degradation ability of Streptomyces sp. D3.

important to understanding the development of artificially beneficial microbial communities to degrade organic contaminant at faster rate.

By culture-dependent method, Pseudomonas and Phyllobacterium were the most frequently obtained bacterial genus, representing percentage $16.7 \%$ and $16.7 \%$ of the isolates, followed by Aminobacter (12.5\%), Pseudoxanthomonas (12.5\%) and Bacillus (12.5\%). However, D3 with high degradation efficiency account for only $4.2 \%$ of the 24 strains. Isolation on YMM containing DDT may have created a selective growth conditions that favored these isolates.

Analysis of the Illumina Miseq data suggested that the diversity of microbial community declined with time. Conversely, the relative abundance of Pseudorhodoferax, Pseudoxanthomonas, Aminobacter, Dokdonell and Hydrogenophaga within the community increased, of which Pseudorhodoferax reached $53.8 \%$ and became the dominant genus. Table 1 and Table 3 show that culture-dependent and -independent methods provided two extremely different compositions of the microbial consortia. The most abundant bacterial OTU Pseudorhodoferax, recovered by sequencing but do not correspond with the isolates obtained via culturing method. Moreover, Pseudomonas was the most frequently isolated by traditional culture method, but only represented the 12th abundant OTU in the culture-independent dataset. Most of the common OTUs belong to the Pseudoxanthomonas and Aminobacter. This implied that the microbes selected by traditional culture-dependent methods are not even representative of those highly competitive species in complex environment. This phenomenon was similar to Stefani et al., (2015) where only a minority of the bacterial OTUs were shared between identification methods in hydrocarbon-contaminated soils, none of the microbial isolates were identified as representatives of the major bacterial OTUs recovered by 454-pyrosequencing. Carraro et al. (2011) also reported similar results during Montasio cheese manufacturing generating different cheese microbial community structures by the culture-dependent approach and the two cultureindependent approaches. The microbes captured by selective culturing method were in low abundance or absent in the sequencing database, this phenomenon indicated that the panorama of microbial community composition in environment couldn't be restored by traditional culture method. Therefore, we need to produce a general view of microbial communities by using culture-independent methods, such as highthroughput sequencing, as well as take into account the limitations associated with culture mediums. While our selective agar was identical to that used for growth in the microcosm, we tried to isolate Pseudorhodoferax and Rhodoferax, but the most abundant bacterial OTU Pseudorhodoferax was not isolated. The development of a more specific medium that is suitable for target strain growth and reproduction will likely have more success in the future.
Streptomyces sp. D3 showed the highest DDT-degrading activity (77\%) after 7 days of incubation, which is higher than most of the DDT-degrading bacteria reported previously. DDT-degrading microorganisms have been reported to mineralize or cometabolize DDT and analogues such as Alcaligenes sp. KK (cometabolize 66.5\% of DDT) (Xie et al., 2011); and Pseudomonas strain 12-3 (degrade up to 51\% DDT in 8 days under liquid culture conditions) (Niu et al., 2012). Streptomyces sp. D3, isolated in this study, is of interest due to its ability to degrade PCB77 and PCNB. The degradation rates were $75 \%$ at a concentration of $20 \mathrm{mg} \mathrm{L}^{-1}$ and $30 \%$ at a concentration of $40 \mathrm{mg} \mathrm{L}^{-1}$, respectively. In comparison to other microorganisms that degrade PCB 77, the degradation rates of strain D3 was relatively high. Recently, Wang et al. (2016b) reported that PCB 77 biodegradation efficiency of Sinorhizobium meliloti NM was $66.9 \%$ at an initial PCB 77 concentration of $1 \mathrm{mg} \mathrm{L}^{-1}$ in 12 days, but the efficiency was only $21.5 \%$ at an initial concentration of $10 \mathrm{mg} \mathrm{L}^{-1}$. In addition, Paenibacillus sp. KBC101 possessed 56\% degradation activity to $10 \mathrm{mg} \mathrm{L}^{-1}$ PCB 77 (Sakai et al., 2005), while Streptomyces sp. D3 showed stronger biodegradation ability than S. meliloti NM and Paenibacillus sp. KBC101 in a short period ( 7 days) at a high concentration (20 $\mathrm{mg} \mathrm{L}^{-1}$ ). In short, D3 showed relatively strong degradation activity toward DDT, PCB77 and PCNB, which maybe attribute to the similar structure of these organochlorine compounds and some common metabolic pathways. Moreover, it is reported that Streptomyces could produce dioxygenas, decarboxylase and hydrolase etc. which could conduct dioxygenation, meta-ring cleavage, hydrolysis reactions to degrade DDT, PCB (Fang et al., 2014).

\section{Conclusions}

The isolate, Streptomyces sp. D3 showed the highest DDT degradation ability and could also metabolize PCB77 and PCNB. The culturedependent and culture-independent methods provide two extremely distinct views of the microbial consortia. By traditional culture method, Pseudomonas was the most frequently isolated (percentage 16.7\%) but only represented the 12 th most abundant OTU in the sequencing dataset (relative abundance $0.9 \%$ ). The most abundant bacterial OTU Pseudorhodoferax (relative abundance 53.8\%) observed by sequencing was not recovered via culturing. This indicated that the traditional culture-dependent methods cannot fully restore the microbial community, thus we should produce a general view of microbial ecology by high-throughput sequencing and obtain degrading microbes purposefully with specific targeted isolation medium. Furthermore, an understanding of the structure and function of microbial community is essential and maybe achieved through the study of artificial microbial communities.

\section{Acknowledgements}

We thank the Key Research Program of the Chinese Academy of Sciences (Grant No. KFZD-SW-303) and the National Natural Science Foundation of China (41671325, U1662110) for financial support.

\section{References}

Åhman, J., Johansson, T., Olsson, M., Punt, P.J., van den Hondel, C.A., Tunlid, A., 2002. Improving the pathogenicity of a nematode-trapping fungus by genetic engineering of a subtilisin with nematotoxic activity. Appl. Environ. Microbiol. 68 (7), 3408-3415.

Andersson, A.F., Riemann, L., Bertilsson, S., 2010. Pyrosequencing reveals contrasting seasonal dynamics of taxa within Baltic Sea bacterioplankton communities. ISME J. 4 171-181.

Bertani, G., 1954. Studies on lysogenesis III. Superinfection of lysogenic Shigella dysenteriae with temperate mutants of the carried phage. J. Bacteriol. 67, 696-707.

Bolan, N., 2012. DDT remediation in contaminated soils: a review of recent studies. Biodegradation 23 (6), 851-863.

Carraro, L., Maifreni, M., Bartolomeoli, I., Martino, M.E., Novelli, E., Frigo, F., Marino, M. Cardazzo, B., 2011. Comparison of culture-dependent and -independent methods for bacterial community monitoring during Montasio cheese manufacturing. Res. Microbiol. 162, 231-239.

Claesson, M.J., O'Sullivan, O., Wang, Q., Nikkila, J., Marchesi, J.R., Smidt, H., de Vos, W.M., Ross, R.P., O'Toole, P.W., 2009. Comparative analysis of pyrosequencing and a 
phylogenetic microarray for exploring microbial community structures in the human distal intestine. PLoS One 4.

Cornforth, D.M., Foster, K.R., 2013. Competition sensing: the social side of bacterial stress responses. Nat. Rev. Microbiol. 11, 285-293.

Dimitriadou, L., Malarvannan, G., Covaci, A., Iossifidou, E., Tzafettas, J., Zournatzi-Koiou, V., Kalantzi, O.I., 2016. Levels and profiles of brominated and chlorinated contaminants in human breast milk from Thessaloniki, Greece. Sci. Total Environ. 539, 350-358.

Eden, P.A., Schmidt, T.M., Blakemore, R.P., Pace, N.R., 1991. Phylogenetic analysis of Aquaspirillum magnetotacticum using polymerase chain reaction-amplified $16 \mathrm{~S}$ rRNA-specific DNA. Int. J. Syst. Evol. Microbiol. 41 (2), 324-325.

Fang, H., Dong, B., Yan, H., Tang, F.F., Yu, Y.L., 2010. Characterization of a bacterial strain capable of degrading DDT congeners and its use in bioremediation of contaminated soil. J. Hazard. Mater. 184, 281-289.

Fang, H., Cai, L., Yang, Y., Ju, F., Li, X.D., Yu, Y.L., Zhang, T., 2014. Metagenomic analysis reveals potential biodegradation pathways of persistent pesticides in freshwater and marine sediments. Sci. Total Environ. 470, 983-992.

Feng, K., Yu, B.Y., Ge, D.M., Wong, M.H., Wang, X.C., Cao, Z.H., 2003. Organochlorine pesticide (DDT and $\mathrm{HCH}$ ) residues in the Taihu Lake Region and its movement in soilwater system I. Field survey of DDT and $\mathrm{HCH}$ residues in ecosystem of the region. Chemosphere 50, 683-687.

Foster, K.R., Bell, T., 2012. Competition, not cooperation, dominates interactions among culturable microbial species. Curr. Biol. 22, 1845-1850.

Giovannoni, S.J., Britschgi, T.B., Moyer, C.L., Field, K.G., 1990. Genetic diversity in Sargasso sea bacterioplankton. Nature 345, 60-63.

Harada, N., Takagi, K., Baba, K., Fujii, K., Iwasaki, A., 2010. Biodegradation of diphenylarsinic acid to arsenic acid by novel soil bacteria isolated from contaminated soil. Biodegradation 21, 491-499.

Hou, J.Y., Liu, W.X., Wang, B.B., Wang, Q.L., Luo, Y.M., Franks, A.E., 2015. PGPR enhance phytoremediation of petroleum contaminated soil and rhizosphere microbial community response. Chemosphere 138, 592-598.

Hu, G.C., Luo, X.J., Dai, J.Y., Zhang, X.L., Wu, H., Zhang, C.L., Guo, W., Xu, M.Q., Mai, B.X., Wei, F.W., 2008. Brominated flame retardants, polychlorinated biphenyls, and organochlorine pesticides in captive giant panda (Ailuropoda melanoleuca) and red pand (Ailurus fulgens) from China. Environ. Sci. Technol. 42, 4704-4709.

Hugenholtz, P., Goebel, B.M., Pace, N.R., 1998. Impact of culture-independent studies on the emerging phylogenetic view of bacterial diversity. J. Bacteriol. 180, 6793

Jiang, H., Dong, H., Zhang, G., Yu, B., Chapman, L.R., Fields, M.W., 2006. Microbial diversity in water and sediment of Lake Chaka, an athalassohaline lake in northwestern China. Appl. Environ. Microbiol. 72 (6), 3832-3845.

Kamanavalli, C.M., Ninnekar, H.Z., 2004. Biodegradation of DDT by a Pseudomonas species. Curr. Microbiol. 48, 10-13.

Kim, Y.M., Ahna, C.K., Wood, S.H., Jungb, G.Y., Parka, J.M., 2009. Synergic degradation of phenanthrene by consortia of newly isolated bacterial strains. J. Bacteriol. 144 293-298.

Kim, J., Sun, Q., Yue, Y., Yoon, K.S., Whang, K.Y., Clark, J.M., Park, Y., 2016. 4, 4'-dichlorodiphenyltrichloroethane (DDT) and 4, 4'-dichlorodiphenyldichloroethylene (DDE) promote adipogenesis in 3T3-L1 adipocyte cell culture. Pestic. Biochem. Physiol. 131, 40-45.

Li, Y.Y., Yang, H., 2013. Bioaccumulation and degradation of pentachloronitrobenzene in Medicago sativa. J. Environ. Manag. 119, 143-150.

Liu, W., Luo, Y., Teng, Y., Li, Z., Ma, L.Q., 2010. Bioremediation of oily sludge-contaminated soil by stimulating indigenous microbes. Environ. Geochem. Health 32, 23-29.

Nadeau, L.J., Menn, F.M., Breen, A., Sayler, G.S., 1994. Aerobic degradation of 1,1,1 trichloro-2,2-bis(4-chlorophenyl)ethane (DDT) by Alcaligenes-eutrophus A5. Appl. Environ. Microbiol. 60, 51-55.

Niu, J., Wang, J., Cui, D., Liu, X., Guang, H., 2012. Study on the isolation, identification and degradation characterisation of a DDT-degrading bacteria. Adv. Mater. Res. 518-523, 2030-2033.
Pieper, D.H., Reineke, W., 2000. Engineering bacteria for bioremediation. Curr. Opin. Biotechnol. 11, 262-270.

Qian, P.Y., Wang, Y., Lee, O.O., Stanley, K L.]->C.K.L., Yang, J.K., Lafi, F.F., Al-Suwailem, A., Wong, T.Y., 2011. Vertical stratification of microbial communities in the Red Sea revealed by $16 \mathrm{~S}$ rDNA pyrosequencing. ISME J. 5, 507-518.

Roesch, L.F., Fulthorpe, R.R, Riva, A., Casella, G Hadwin, A.K, Kent, A.D. Daroub, S.H. Camargo, F.A., Farmerie, W.G., Triplett, E.W., 2007. Pyrosequencing enumerates and contrasts soil microbial diversity. ISME J. 1 (4), 283-290.

Sakai, M., Ezaki, S., Suzuki, N., Kurane, R., 2005. Isolation and characterization of a novel polychlorinated biphenyl-degrading bacterium, Paenibacillus sp. KBC101. Appl. Microbiol. Biotechnol. 68, 111-116.

Shade, A., Hogan, C.S., Klimowicz, A.K., Linske, M., McManus, P.S., Handelsman, J., 2012. Culturing captures members of the soil rare biosphere. Environ. Microbiol. 14, 2247-2252.

Shi, Y., Meng, F., Guo, F., Lu, Y., Wang, T., Zhang, H., 2005. Residues of organic chlorinated pesticides in agricultural soils of Beijing, China. Arch. Environ. Contam. Toxicol. 49, 37-44.

Simonich, S.L., Hites, R.A., 1995. Global distribution of persistent organochlorine compounds. Science 269 (5232), 1851

Sivasubramaniam, D., Franks, A.E., 2016. Bioengineering microbial communities: their potential to help, hinder and disgust. Bioengineering 7 (3), 137-144.

Stefani, F.O., Bell, T.H., Marchand, C., Ivan, E., El Yassimi, A., St-Arnaud, M., Hijri, M., 2015. Culture-dependent and-independent methods capture different microbial community fractions in hydrocarbon-contaminated soils. PLoS One 10 (6), e0128272.

Sudharshan, S., Naidu, R., Mallavarapu, M., Bolan, N., 2012. DDT remediation in contaminated soils: a review of recent studies. Biodegradation 23 (6), 851-863.

Takagi, K., Iwasaki, A., Kamei, I., Satsuma, K., Yoshioka, Y., Harada, N., 2009. Aerobic mineralization of hexachlorobenzene by newly isolated pentachloronitrobenzenedegrading Nocardioides sp. strain PD653. Appl. Environ. Microbiol. 75, 4452-4458.

Vaz-Moreira, I., Egas, C., Nunes, O.C., Manaia, C.M., 2011. Culture-dependent and cultureindependent diversity surveys target different bacteria: a case study in a freshwater sample. Antonie Van Leeuwenhoek 100, 245-257.

Verma, J.P., Jaiswal, D.K., Sagar, R., 2014. Pesticide relevance and their microbial degradation: a-state-of-art. Rev. Environ. Sci. Biotechnol. 13, 429-466.

Wang, G.L., Bi, M., Liang, B., Jiang, J.D., Li, S.P., 2011. Pseudoxanthomonas jiangsuensis sp. Nov., a DDT-degrading bacterium isolated from a long-term DDT-polluted soil. Curr. Microbiol. 62, 1760-1766.

Wang B. Wu, C., Liu, W., Teng, Y., Luo, Y., Christie, P., Guo, D., 2016a. Levels and patterns of organochlorine pesticides in agricultural soils in an area of extensive historical cotton cultivation in Henan province, China. Environ. Sci. Pollut. Res. 23, 6680-6689.

Wang, X., Teng, Y., Luo, Y., Dick, R.P., 2016b. Biodegradation of 3, 3', 4, 4'tetrachlorobiphenyl by Sinorhizobium meliloti NM. Bioresour. Technol. 201, 261-268.

World Health Organization, 1989. DDT and Its Derivatives: Environmental Aspects. Vol. 9. World Health Organisation, Geneva.

Xie, H., Zhu, L.S., Xu, Q.F., Wang, J., Liu, W., Jiang, J.H., Meng, Y., 2011. Isolation and degradation ability of the DDT-degrading bacterial strain KK. Environ. Earth Sci. 62, 93-99.

Ye, L., Zhang, T., 2013. Bacterial communities in different sections of a municipal wastewater treatment plant revealed by $16 \mathrm{~S}$ rDNA 454 pyrosequencing. Appl. Microbiol. Biotechnol. 97, 2681-2690.

Zhong, G.C., Tang, J.H., Zhao, Z., Pan, X.H., Chen, Y.J., Li, J., Zhang, G., 2011. Organochlorine pesticides in sediments of Laizhou Bay and its adjacent rivers, North China. Mar. Pollut. Bull. 62, 2543-2547.

Zhou, J., Wu, L., Deng, Y., Zhi, X., Jiang, Y.-H., Tu, Q., Xie, J., Van Nostrand, J.D., He, Z., Yang, Y., 2011. Reproducibility and quantitation of amplicon sequencing-based detection. ISME J. 5 (8), 1303-1313. 